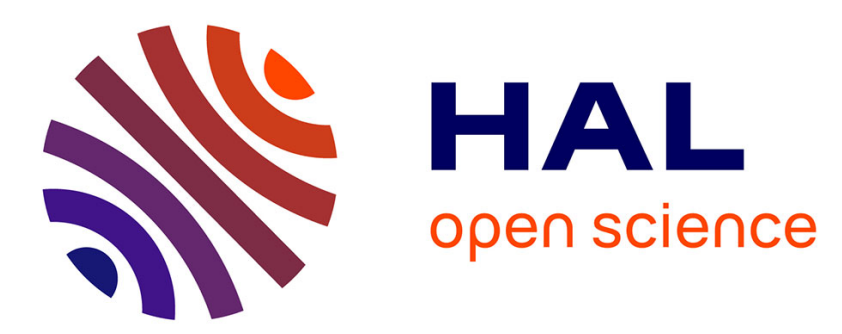

\title{
Charles Stépanoff, Carole Ferret, Gaëlle Lacaze, Julien Thorez, Nomadismes d'Asie centrale et septentrionale Irène Mestre
}

\section{To cite this version:}

Irène Mestre. Charles Stépanoff, Carole Ferret, Gaëlle Lacaze, Julien Thorez, Nomadismes d'Asie centrale et septentrionale. Géocarrefour - Revue de géographie de Lyon, 2015, 90 (3), 10.4000/geocarrefour.9123 . hal-01907294

\section{HAL Id: hal-01907294 \\ https://hal.science/hal-01907294}

Submitted on 12 Nov 2018

HAL is a multi-disciplinary open access archive for the deposit and dissemination of scientific research documents, whether they are published or not. The documents may come from teaching and research institutions in France or abroad, or from public or private research centers.
L'archive ouverte pluridisciplinaire HAL, est destinée au dépôt et à la diffusion de documents scientifiques de niveau recherche, publiés ou non, émanant des établissements d'enseignement et de recherche français ou étrangers, des laboratoires publics ou privés. 


\section{Géocarrefour}

\section{Charles Stépanoff, Carole Ferret, Gaëlle Lacaze, Julien Thorez, Nomadismes d'Asie centrale et septentrionale}

Irène Mestre

\section{(2) OpenEdition}

Journals

Édition électronique

URL : http://journals.openedition.org/geocarrefour/9123

ISSN : 1960-601X

\section{Éditeur}

Association des amis de la Revue de géographie de Lyon

Édition imprimée

Date de publication : 2 décembre 2015

Pagination : 282

ISSN : 1627-4873

Ce document vous est offert par BU de l'Université Jean Moulin Lyon 3

\section{JEAN MOULIN}

\section{Référence électronique}

Irène Mestre, «Charles Stépanoff, Carole Ferret, Gaëlle Lacaze, Julien Thorez, Nomadismes d'Asie centrale et septentrionale », Géocarrefour [En ligne], 90/3 | 2015, mis en ligne le , consulté le 29 octobre 2018. URL : http://journals.openedition.org/geocarrefour/9123

Ce document a été généré automatiquement le 29 octobre 2018.

(C) Géocarrefour 


\title{
Charles Stépanoff, Carole Ferret, Gaëlle Lacaze, Julien Thorez, Nomadismes d'Asie centrale et septentrionale
}

\author{
Irène Mestre
}

\section{RÉFÉRENCE}

STÉPANOFF C., FERRET C., LACAZE G., THOREZ J. (dir.), 2013, Nomadismes d'Asie centrale et septentrionale, Paris, Armand Colin, $287 \mathrm{p}$.

1 Avec ce beau livre richement illustré, les auteurs offrent une description générale des différents nomadismes et explorent de manière transversale les facteurs de leur adaptation et de leur continuité. L'équipe transdisciplinaire à l'origine de l'ouvrage est composée d'anthropologues, de géographes, d'historiens et d'archéologues et compte 42 auteurs. Elle est le fruit de la collaboration originale d'établissements français, kirghizistanais et russes.

2 Longtemps perçu comme archaïque, le nomadisme n'est plus considéré comme une étape transitoire vers la sédentarisation depuis les années 1990. Ce tournant est dû à reconnaissance grandissante de son rôle pour la protection de l'environnement (Ciadella et. al., 2010) et à la revalorisation de ce mode de vie. Les auteurs s'attachent à faire découvrir la complexité des populations nomades et anciennement nomades sur un vaste territoire qui s'étend de la mer Caspienne à l'océan Pacifique, bordé au nord par l'océan glacial arctique, comprenant au sud le Tadjikistan et le nord de la Chine. Les espaces et populations étudiés sont liés par l'expérience du socialisme et, pour partie, par celle des transformations postsoviétiques. Les auteurs prennent en compte la variété des trajectoires des populations nomades qui combinent activités de production en adaptant 
leurs déplacements. La définition du nomadisme n'est pas établie selon les spécificités habituelles, pratique du pastoralisme ou appartenance à certains groupes ethniques, mais selon un ensemble de critères qui comprennent les déplacements effectués en famille, le lien étroit avec les animaux dont les besoins et l'accessibilité guident en partie les déplacements et le « [...] lien paradoxal à l'espace fait d'une conjonction de maîtrise et de retrait» (p.8). La variété de l'objet d'étude est soulignée par la typologie des modes d'habitat mobile depuis le déplacement de groupes jusqu'à celui des bergers professionnels (p. 11).

3 Basé sur des études de cas et des données de terrain, l'ouvrage est divisé en cinq parties. Dans la première partie, les caractéristiques des populations à l'échelle de leur écosystème sont étudiées en se penchant sur les savoirs, les systèmes mobiles et les techniques de déplacement. La deuxième partie traite des périodes de non-mobilité au sein de l'habitation, du campement et des relations entre groupes et ménages, en insistant sur le paradoxe entre l'usage mobile des ressources naturelles et l'ancrage dans le territoire. La troisième partie aborde l'individu à travers l'apprentissage social et les axes structurants de la perception nomade du monde. La quatrième partie est consacrée aux relations entre les hommes et les animaux, à travers les activités de production telles que l'élevage, la chasse, la pêche ainsi qu'à travers les relations aux animaux en dehors des cycles de production. La dernière partie s'interroge sur les relations entre populations nomades et sédentaires en s'appuyant sur une approche historique à partir de l'Age du bronze. Depuis les mouvements de sédentarisation aux époques tsariste et soviétique jusqu'aux indépendances, la place du nomadisme a évolué dans un espace en mutation où l'urbanisation et l'économie industrielle progressent. Les auteurs construisent une analyse riche sur la relation entre les populations nomades et la politique, qui est le plus souvent le fait de populations sédentaires. Les différences entre les nouvelles républiques d'Asie centrale et la Chine sont mises en lumière dans leur manière d'aborder la question de l'héritage nomade (p. 271 et 273). En Chine, l'opposition entre les Evenks et les touristes citadins s'exprime à travers les activités touristiques contrôlées par les autorités. A l'inverse, au Kazakhstan, le nomadisme est un pilier de la construction de la nouvelle identité nationale qui se traduit par la politique de développement $d u$ tourisme écologique et ethnique, comme vitrine vers les pays extérieurs, et par la référence à l'identité nomade dans l'aménagement de la nouvelle capitale (p. 276).

4 La richesse des cartes et des schémas de localisation (p. 101), de déplacements (p. 66 et 189), de variation altitudinale des campements (p.67), de saisonnalité des activités d'élevage (p. 171 et 194) permet de situer dans le temps et dans l'espace les différents aspects de la vie des populations nomades. De même, les photographies, principalement des auteurs, occupent une place importante et soutiennent judicieusement l'argumentaire. Les encadrés disposés à intervalles réguliers donnent des indications sur des points complémentaires comme par exemple les techniques d'abattages du bétail ou la fabrication du feutre.

5 Les questions de transmission des savoirs, d'accès aux ressources naturelles et du rôle des envois de fonds de la part des migrants abordées de manière transversale sont des facteurs sociaux et économiques qui ouvrent des perspectives pour une analyse des capacités d'adaptation de ces systèmes. Dans une perspective géographique, les deux apports les plus importants sont l'élaboration de nouvelles typologies et le croisement d'échelles. 
6 En premier lieu, l'approche systémique du nomadisme permet partiellement de dépasser les spécificités des cas d'études. La typologie basée sur la composition du cheptel (p. 170) souligne la variété des profils et leur distribution spatiale. Les cheptels où les rennes, les ovins, les caprins et les chameaux sont plus importants que dans le profil moyen sont les plus courants dans les populations nomades. De même, si ces troupeaux ne concentrent pas la majorité du bétail de la zone, ils couvrent près de la moitié du territoire. Les auteurs déconstruisent la frontière entre sédentaires et nomades pour souligner les connexions existantes (p.230) qui sont envisagées d'un point de vue des déplacements (p. 61 et 64), de la structuration des espaces (p. 99) et des échanges marchands (p. 265). Les exemples des héritages multiformes des mondes nomades illustrent l'intégration réciproque des deux modes de pensées. Cependant, l'analyse ne cache pas la complémentarité et les oppositions (p. 256) qui revêtent des aspects politiques (p. 267).

7 En deuxième lieu, le rôle des échelles est mis en avant par la présentation des trois premières parties qui passent successivement du macro au méso et au micro. En plus de servir de trame descriptive, cette grille multiscalaire nourrit l'analyse sur la dynamique des typologies en montrant les écarts entre trajectoires personnelles et collectives. Dans le cas de la Bouriatie (p. 248), à la suite des processus de privatisation, les ménages ont reçu des pâturages dont la capacité de charge est plus faible que celle des pâturages utilisés collectivement auparavant. De plus, les pâturages les plus proches ont été favorisés et les chemins de transhumance vers les pâturages éloignés sont devenus inaccessibles pour des raisons de droits d'accès. Alors qu'à l'échelle du ménage, ces évolutions permettent au nouveau mode d'exploitation d'être viable, en cas de catastrophe climatique la réduction de la mobilité et donc de la flexibilité menacerait le système à l'échelle collective.

8 Alors que les auteurs répondent à la tâche descriptive des populations nomades, la question de l'adaptation aurait appelé une conclusion. Néanmoins, la très grande diversité présentée et le croisement des disciplines tracent des pistes pour une meilleure compréhension des populations nomades en proposant des axes de lecture plutôt qu'un discours englobant.

Par son langage clair comme par le choix des illustrations ce livre permet une double lecture: il convainc les chercheurs par la solidité des raisonnements ainsi que par la définition systémique des nomadismes et, il donne aux lecteurs non-spécialistes des explications accessibles sur les modes de vie des populations nomades. Il résonne pour tous comme un appel au voyage.

\section{BIBLIOGRAPHIE}

CIALDELLA N., HOSTIOU N., GIRARD N., 2010, Quels liens entre élevages et écosystèmes naturels?, Natures Sciences Sociétés, 1 mars, vol. 18, n¹, p. 24-35. 